\title{
Forecast for the Cameroon's Residential Electricity Demand Based on the Multilinear Regression Model
}

\author{
Serge Guefano' ${ }^{1}$ Jean Gaston Tamba1, Louis Monkam¹, Beguide Bonoma ${ }^{2}$ \\ ${ }^{1}$ Institute of Technology, University of Douala, Douala, Cameroon \\ ${ }^{2}$ Higher Teacher Training College, University of Yaoundé I, Yaoundé, Cameroon \\ Email: sergebenoitbidimbo@gmail.com
}

How to cite this paper: Guefano, S., Tamba, J.G., Monkam, L. and Bonoma, B. (2020) Forecast for the Cameroon's Residential Electricity Demand Based on the Multilinear Regression Model. Energy and Power Engineering, 12, 182-192.

https://doi.org/10.4236/epe.2020.125013

Received: February 26, 2020

Accepted: May 18, 2020

Published: May 21, 2020

Copyright () 2020 by author(s) and Scientific Research Publishing Inc. This work is licensed under the Creative Commons Attribution International License (CC BY 4.0).

http://creativecommons.org/licenses/by/4.0/

\begin{abstract}
The electricity needs of populations in Cameroon are increasing and are still very inadequate. Companies, public buildings and households are facing frequent blackout which constrain development and social well-being. Therefore, the present work tried to forecast the electricity demand in the residential sector in Cameroon, in order to contribute significantly to the mastery of electricity consumption and highlight decision-makers in this sector. Six macroeconomics parameters covering the period 1994-2014 are used for these issues. Stationarity tests within gross domestic product, gross domestic product per capita, electricity consumption, population and numbers of subscribers and households respectively; reveal that all the series are $I(1)$. Thus, the VAR (Vector Autoregressive) model has been retained to forecast the electricity demand until 2020. The cusum test and the cusum of squared test attest the stability of that model with a margin of error of $0.02 \%$. Previsions are then more reliable and show that the electric request will skip from 1721 GWh in 2014 to more than 2481 GWh in 2020 approximatively, following a growing yearly rate of $5.36 \%$. In order to reach its emergence, Cameroon ought to speed up its production in the domain of hydroelectric and thermal grid in order to meet the requirements in electric power in short and long term.
\end{abstract}

\section{Keywords}

Modeling of the Electricity Demand, Forecast, Residential Sector, VAR Model, Cameroon

\section{Introduction}

Electricity is the main factor of economic growth in the world and in Cameroon 
in particular [1]. Its relative importance increases with the progress in technics, industrialization and the need of modern convenience [2]. The articulations of the document of strategy for growth and employment show clearly that Cameroon inexorably comes within the scope of policy of development omnidirectional of sectors of activities. This development is without ambiguity subjected to a large demand of electricity [3]. The electric supply has therefore to be situated near the demand.

Unfortunately, in our country, the situation is different. Despite the country is the second hydroelectric potential owner in sub-Saharan Africa after the Democratic Republic of Congo, the lack of electricity in our industries causes the loss of production of more the 12 million USD per year [4].

The rate of access to electricity in the country is around $49 \%$ for the households in the urban areas. More than the half residences remain in darkness. Such a situation is also observed in rural areas where the major part of residences does not have access to electric networks [5] [6].

Those timeworn and saturated infrastructures cause the loss of more than $30 \%$ of the total production [5]. The main part production of electricity is then lost whereas the population needs in energy are increasing [7]. Thus, the electric demanding system appears as a necessity to study, in the double objective of identifying the different determinants and bringing out their respective influence on the consumption. Some researches are carried out in that perspective. Fotsing et al., carried out an analysis of demand and supply of electricity energy in Cameroon, and demonstrated the impact of meteorological parameters on the monthly power peak of South and North interconnected electricity networks [8]. These same authors did a research on influence of socio-economic indicators on electricity consumption of low voltage customers in Cameroon. Here, data of the electricity consumption on low voltage are considered as proxy of the electricity consumption of the residential sector in Cameroon, and are used to model the electricity demand of that sector using ARMA, ARIMA, SARIMA and Coob-Douglas models, but with no forecasting [9].

For these reasons, the present paper highlights modeling and forecasting electricity demand in Cameroon with a wide range of parameters. The electricity consumption, the gross domestic product, the gross domestic product per capita, population, the numbers of households and subscribers are the main variables to take into account in this work. The residential sector targeted in our work is made up of $71 \%$ of households [9], 29\% of small and medium sized enterprises (SMSE) small and medium industries (SMI), schools and universities, hospitals and offices whose daily values of electricity consumption are under 400 Watts [10]. Such an initiative would give opportunity to scientifically follow the decisions of the different actors of the domain, the various projects related to hydroelectric and thermal infrastructural development; and to get in view the possible trends in electricity need, if one of the macroeconomic parameters had suddenly fluctuated. This paper is subdivided in four mains sections: the section two deals with the adopted econometric method and the description of data, the 
third section focuses on results. In the fourth and last section, we drawn-on a conclusion from the proposed analysis.

\section{Econometric Method and Data Description}

\subsection{Econometric Method}

The multilinear regression is a formalized representation of phenomena under equations that variables are economic magnitudes. If one indicates $y$ the electricity consumption in lower voltage and considered as proxy for the electricity demand of the residential sector, $x_{p} ; x_{p h}, x_{p o}, x_{m}$ and $x_{a}$ the respective representations of the Gross domestic product, gross domestic product per capita, the population, the numbers of households and subscribers; consumption $y(t)$ on a date $t$ is described by the multilinear form (Equation (1)).

$$
y(t)=a_{0}+a_{1} x_{p}(t)+a_{2} x_{p h}(t)+a_{3} x_{P_{O}}(t)+a_{4} x_{m}(t)+a_{5} x_{a}(t)+\varepsilon_{t}
$$

In this equation, $y$ is the endogenous, the $x$ variables are exogenous, $a_{i}, i \in[1,5]$ are the coefficients of the model, $a_{0}$ the constant term and $\varepsilon_{t}$ for $t=1,2, \cdots, n$ represents the residues within the model and $n$ the number of observations. But those coefficients are not generally known and must be estimated for that reason. In this case, the model in Equation (1) takes the form given by (Equation (2)).

$$
\hat{y}(t)=\hat{a}_{0}+\hat{a}_{1} x_{p}(t)+\hat{a}_{2} x_{p h}(t)+\hat{a}_{3} x_{P o}(t)+\hat{a}_{4} x_{m}(t)+\hat{a}_{5} x_{a}(t)
$$

The residues $\varepsilon_{t}$ are obtained by making the difference between the real model and the estimated one as the relation indicated in Equation (3).

$$
\varepsilon_{t}=y(t)-\hat{y}(t)
$$

A set of statistical tests must be carried out in order to test the relevance of such a model, such as the test of unit root on which we will focus first.

\subsubsection{Unit Root Test}

We mainly find here, the Dickey Fuller (DF) test, Augmented Dickey Fuller (ADF) test and the Phillips-Peron test, which rest on the estimation with ordinary least square of the tree equations system (4).

$$
\left\{\begin{array}{l}
\Delta y_{t}=\rho y_{t-1}-\sum_{j=2}^{p} \Phi_{j} \Delta y_{t-j+1}+\varepsilon_{t} \\
\Delta y_{t}=\rho y_{t-1}-\sum_{j=2}^{p} \Phi_{j} \Delta y_{t-j+1}+c+\varepsilon_{t} \\
\Delta y_{t}=\rho y_{t-1}-\sum_{j=2}^{p} \Phi_{j} \Delta y_{t-j+1}+b t+c+\varepsilon_{t}
\end{array}\right.
$$

These tests give room to detect all non-stationarity within each observation and to correct it. We agree the hypothesis of non-stationarity of parameter when the associated $\mathrm{P}$-value is above the critical point $\alpha=5 \%$ to evaluate the relevance of the test carried out. In this case, it will be necessary to make multiple differences so as to stabilize it, by calculating quantities $\Delta x(t)$ defined by Equ- 
ation (5).

$$
\Delta x(t)=x(t)-x(t-1)
$$

If at the end of these tests, we have one level variables stationary, they refer $I(0)$. If on the other hand, others are stationary after a first degree difference, they refer to $I(1)$. Hence, if all variables are $I(1)$, the modeling by Vector Autoregressive (VAR) model is possible.

\subsubsection{Modeling VAR}

The VAR multilinear model formulated in 1980 by Christopher Sims is a generalization of ARMA models formulated in their turn by Box-Jenkins in 1978 [11] [12]. Here, the electricity consumption on time $t$ is specified under Equation (6) in accordance with the past values $y(t-i)$ and of residues $\varepsilon(t-i)$.

$$
y(t)=\sum_{i=1}^{p} \alpha_{i} y(t-i)+\sum_{i=1}^{q} \beta_{i} \varepsilon(t-i)+v_{t}
$$

This Equation is a linear form where electricity consumption is depending only on its former values. Christopher Sims generalizes this latter relation under Equation (7), where the consumption $y(t-i)$ depends now on the former values $y(t-i)$ and those of the gross domestic product (GPD) $x_{p}(t-i)$ for instance.

$$
y(t)=a_{1}^{0}+\sum_{i=1}^{z} a_{1 i}^{1} y(t-i)+\sum_{i=1}^{z} a_{1 i}^{2} x_{p}(t-i)+v_{t}
$$

By generalizing this last relation, the model in Equation (7) takes the form given by Equation (8) where the residues $v_{P}$ are considering as white noise.

$$
\begin{aligned}
y(t)= & a_{1}^{0}+\sum_{i=1}^{z}\left[a_{1 i}^{1} y(t-i)+a_{1 i}^{2} x_{p}(t-i)+a_{1 i}^{3} x_{p h}(t-i)\right] \\
& +\sum_{i=1}^{z}\left[a_{1 i}^{4} x_{p o}(t-i)+a_{1 i}^{5} x_{m}(t-i)+a_{1 i}^{6} x_{a}(t-i)\right]+v_{P}
\end{aligned}
$$

If the lag number $P$ retained by the test is one, the use of electricity at a period $t$ would depend on all period shifted variables. In that case, Equation (8) takes the form given by Equation (9).

$$
\begin{aligned}
y(t)= & a_{1}^{0}+a_{11}^{1} y(t-1)+a_{11}^{2} x_{p}(t-1)+a_{11}^{3} x_{p h}(t-1) \\
& +a_{11}^{4} x_{p o}(t-1)+a_{11}^{5} x_{m}(t-1)+a_{11}^{6} x_{a}(t-1)+v_{p}
\end{aligned}
$$

If a first-degree difference is necessary to stabilize each parameter, then the precedent Equation takes the place of (Equation (10)).

$$
\begin{aligned}
\Delta y(t)= & a_{1}^{0}+a_{11}^{1} \Delta y(t-1)+a_{11}^{2} \Delta x_{p}(t-1)+a_{11}^{3} \Delta x_{p h}(t-1) \\
& +a_{11}^{4} \Delta x_{p o}(t-1)+a_{11}^{5} \Delta x_{m}(t-1)+a_{11}^{6} \Delta x_{a}(t-1)+v_{p}
\end{aligned}
$$

Such a model must then be validated statistically before any test of prevision.

\subsubsection{Validation of the Model}

For the statistical validation of the model specified by Equation (10), we carry out two tests of greater significativity: the test of normality of the residues based 
on Jarque-Bera's $(J B)$ statistic by Equation (11) and the test on the stability model due to the cumulated sum of recursive residues $S(t)$ and the Cumulated sum of the squares of the recursive residues $S^{\prime}(t)$ defined by the formulas (a) and (b) of Equation (12).

$$
J B=\frac{n}{6} \beta_{1}+\frac{n}{24}\left(\beta_{2}-3\right)^{2}
$$

$\beta_{1}$ and $\beta_{2}$ are coefficients of Skewness and Kurtosis respectively.

$$
\left\{\begin{array}{l}
S(t)=(n-k) \frac{\sum_{j=k+1}^{t} \tilde{\varepsilon}_{j}}{\sum_{j=k+1}^{n} \tilde{\varepsilon}_{j}} \\
S^{\prime}(t)=\frac{\sum_{j=k+1}^{t} \tilde{\varepsilon}_{t}^{2}}{\sum_{j=k+1}^{n} \tilde{\varepsilon}_{j}^{2}}
\end{array}\right.
$$

Indeed, the model is statistically stable when the growth of each of the sum is included in the confidential interval $\beta= \pm 95 \%$ of a critical significance size $\alpha= \pm 5 \%$.

Once the stability of the formulated model is proven, any task of prevision is now on possible.

\subsubsection{The Forecast}

In a situation where parameters are stationary after a first-degree difference, the forecasting difference approach can be adopted in order to calculate sequentially, the future values of consumption. We consequently use the relation given by Equation (13).

$$
\hat{y}_{t}=\Delta y_{t}+y_{t-1}
$$

where $\hat{y}_{t}$ is the estimated electricity consumption at the date $t, y_{t-1}$ the real value of consumption at the date $t-1$, and $\Delta y_{t}$ represents electricity consumption gap among the consecutive dates $t$ and $t-1$, and given by the computational software EViews.

The related error to each estimation is given by Equation (14) and the medium error by Equation (15).

$$
\begin{gathered}
\lambda_{t}=1-\frac{\hat{y}_{t}}{y_{t}} \\
\bar{\lambda}=\frac{\sum_{t=1}^{n} \lambda_{t}}{n}
\end{gathered}
$$

\subsection{Data Description}

Data relating to the six parameters used to characterize the electricity demand of the residential sector are collected next to several institutions, and cover the period running from 1994 to 2014. Information to electricity consumption and 
number of subscribers are provided by Energy of Cameroon (ENEO) [13]. Data bases obtained from the World Bank provided information about the evolution of GDP, GDP per capita and population [14]. Data about number of households are provided by Cameroon's Electricity Sector Regulatory Agency (ARSEL) [15]. The link between the electricity consumption and each exogenous seems rather close. Indeed, for each couple: consumption-GDP, consumption-GDP per capita, consumption-population, consumption-number of households, consumption-number of subscribers; correlative coefficients are rated at about $98.2 \%$, 96.27\%, 99.39\%, 98.45\% and 96.44\% respectively. Endogenous consumption seems thus strongly correlated to each of the exogenous ones.

Moreover, when the low voltage consumption grows over all the period of observation according to annual medium rate growth (AMRG) of $5.72 \%$, the economic growth characterized by the GDP follows over the same period, a AMRG of $6.01 \%$, the number of households connected to the electrical network supply follows a AMRG of $2.89 \%$, and the number of ENEO-subscribers moves following a AMRG of $4.11 \%$.

The necessary statistical analysis carried out on EViews, this due to the fact that there is a software of Econometric choice, giving access to a powerful statistical tool of modeling and forecast.

\section{Results and Discussion}

\subsection{Unit Root Tests}

Table 1 recapitulates the results of ADF and PP tests on the search for a unit root within each level variable.

One notes that the P-value associated to the ADF and PP tests on the consumption parameter $y$ is higher than the critical grade $\alpha=5 \%$. Thus, we accept the null assumption, it exists a unitary root, this parameter is not stationary at level point [12]. Somewhere else, the exogenous variables present $\mathrm{P}$-values higher to the fixed critical point. They are not level stationaries; a first-degree difference is therefore used in order to ensure their stationarity.

Table 2 gives results of ADF and PP tests following the model with tendency on differentiated variables.

Table 1. Unit root tests results.

\begin{tabular}{|c|c|c|c|c|}
\hline \multirow{4}{*}{ Variables at level } & F tests & & \multicolumn{2}{|c|}{ PP tests } \\
\hline & \multicolumn{4}{|c|}{ Model with tendency } \\
\hline & \multicolumn{4}{|c|}{$5 \%$ significance } \\
\hline & t-statistic & P-value (\%) & t-statistic & P-value (\%) \\
\hline$y$ & -3.658 & 20.1 & -3.658 & 2.06 \\
\hline$X_{p}$ & -3.658 & 72.65 & -3.661 & 72.64 \\
\hline$X_{p h}$ & -3.733 & 26.25 & -3.658 & 78.57 \\
\hline$X_{p o}$ & -3.733 & 42.37 & -3.658 & 100 \\
\hline$X_{m}$ & -3.658 & 56.6 & -3.658 & 56.66 \\
\hline$X_{a}$ & -3.691 & 99.84 & -3.658 & 99.98 \\
\hline
\end{tabular}


Table 2. Unit root tests on differentiated values.

\begin{tabular}{ccccc}
\hline \multicolumn{2}{c}{ ADF tests } & \multicolumn{2}{c}{ PP tests } \\
\hline & \multicolumn{4}{c}{ Model with tendency } \\
\cline { 2 - 5 } Differentiated Values & \multicolumn{4}{c}{$5 \%$ significance } \\
\cline { 2 - 5 } & t-statistic & P-value (\%) & t-statistic & P-value (\%) \\
\cline { 2 - 5 }$\Delta y$ & -4.518 & 2.06 & -3.658 & 2.06 \\
$\Delta x_{p}$ & -4.489 & 1.09 & -3.661 & 1.06 \\
$\Delta x_{p h}$ & -4.239 & 1.75 & -4.504 & 1.06 \\
$\Delta x_{p o}$ & - & - & -5.121 & 0.32 \\
$\Delta x_{m}$ & -3.825 & 3.77 & -3.807 & 3.92 \\
$\Delta x_{a}$ & -5.095 & 0.38 & -8.639 & 0.0001 \\
\hline
\end{tabular}

It is observed that the associated $\mathrm{P}$-values to each test are respectively lower to the critical point $\alpha=5 \%$. We reject the null hypothesis; all the exogenous variables seem thus been stationary after the first-degree difference and are noted $I(1)$. The differentiated forms: $\Delta y, \Delta x_{p}, \Delta x_{p h}, \Delta x_{p o}, \Delta x_{m}$ and $\Delta x_{a}$ can be retained to model the electricity demand of residential sector in a multilinear representation $\operatorname{VAR}(p)$ where $p$ is this the appropriated lag number.

\subsection{Lag Number}

The appropriate lag number is the one minimizing the maximum of criteria of selection. The statistical tests reveal that the model formulated with differentiated variables, minimizes for a lag number $p=1$, the Akaike, Schwarz, Hannan Quin and Lagrange's criteria; that is four criteria among the five retained by the software.

The electricity consumption can thus be represented by a VAR(1), of order 1 , where each variable is shifted of a period as shown in Equation (10).

\subsection{Estimation of Coefficients of the Model}

Coefficients of model are estimated on EViews with a lag number $p=1$. Equation (10) takes the ultimate's form presented by Equation (16).

$$
\begin{aligned}
\Delta y(t)= & -54.70328+0.074178 \Delta y(t-1)-0.064459 \Delta x_{p}(t-1) \\
& +1.271670 \Delta x_{p h}(t-1)+0.000268 \Delta x_{p o}(t-1) \\
& +0.090435 \Delta x_{m}(t-1)+0.438326 \Delta x_{a}(t-1)+v_{t}
\end{aligned}
$$

\subsection{Validation of the Model}

The tests of validation are realized in order to evaluate the robustness of the specified model.

\subsubsection{The Normality of the Residues Test}

Jarque-Bera's $(J B)$ test gives the possibility to evaluate the normality of residues. Results prove that $J B=0.96$ is quite lower than 5.99. The associated P-value to the test is equivalent to $61.67 \%$ and is higher to the selected critical point. The 
terms of error $v_{t}$ constitute a Gaussian's white noise. All the possible disturbances are independent, normally and identically distributed within the model designed by Equation (16).

\subsubsection{The Stability of the Model Test}

Figure 1 and Figure 2 show that the cumulated sum of recursive residues $S(t)$ and the Cumulated sum of the squares of the recursive residues $S^{\prime}(t)$ are included in the confidential interval $\beta= \pm 95 \%$ to the significant point $\alpha=5 \%$.

The formulated model is statistically valid and can be taken in any exercise of modeling and forecasting in the electricity demand of a residential Sector.

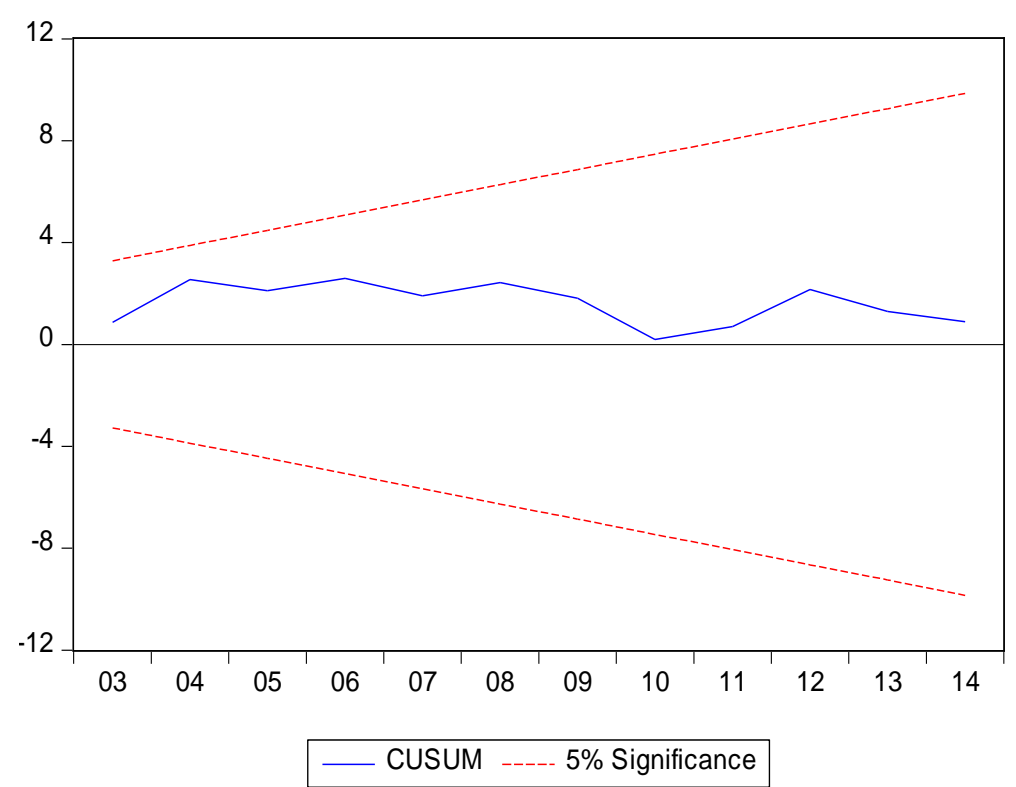

Figure 1. Evolution of the cumulated sum of recursive residues $S(t)$.

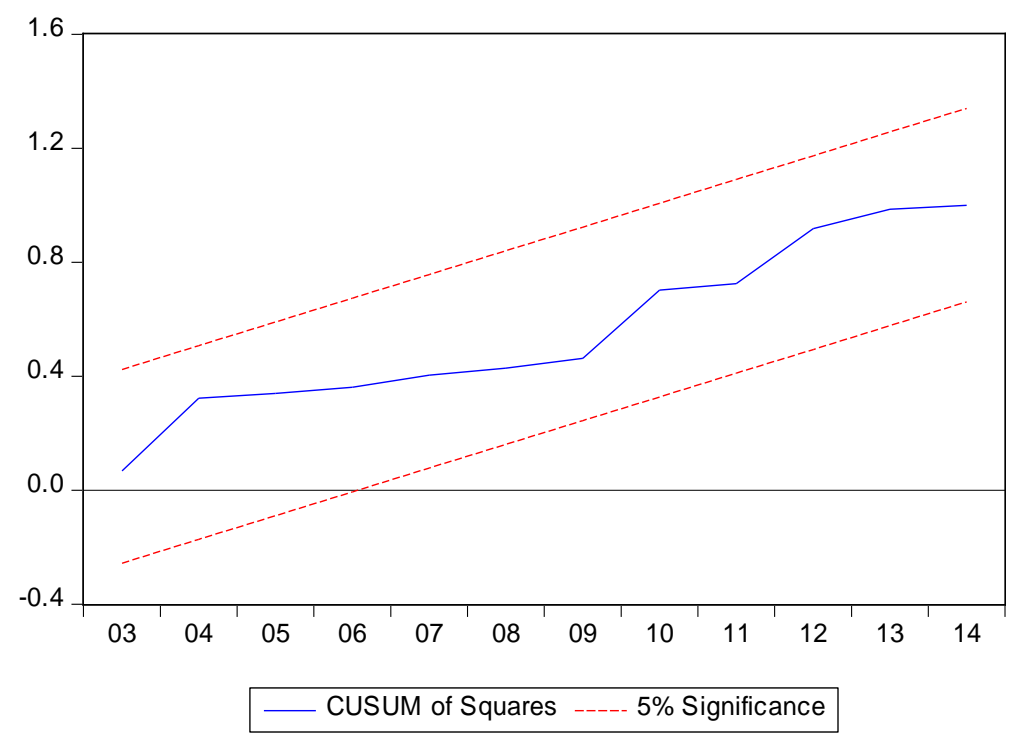

Figure 2. Evolution of the cumulated sum of the squares of the recursive residues $S^{\prime}(t)$. 
Fitted values with this model have to be stable and minimized margin of errors.

Figure 3 shows that the simultaneous growth of real data of consumption in Serie $y$ and those by the model in Serie $y_{p}$ fit one another perfectly.

The model is quite reliable and makes possible to find past consumption values with a medium error of $0.02 \%$. It is thus retained for the forecasting.

\subsection{Forecasting}

Table 3 shows that the residential electricity consumption will skip from 1721 GWh in 2014 to more than 2481 GWh in 2020 following an AMRG of 5.36\%. Figure 4 shows the tendency of electricity consumption between 2014 and 2020. Energy demand in residential sector will remain in full growing in Cameroon, under influence of its main determinants.

Table 3. Electricity consumption forecasting.

\begin{tabular}{cc}
\hline Years & Forecasted values in GWh \\
\hline 2015 & 1781 \\
2016 & 1924 \\
2017 & 2056 \\
2018 & 2192 \\
2019 & 2335 \\
2020 & 2481 \\
\hline
\end{tabular}

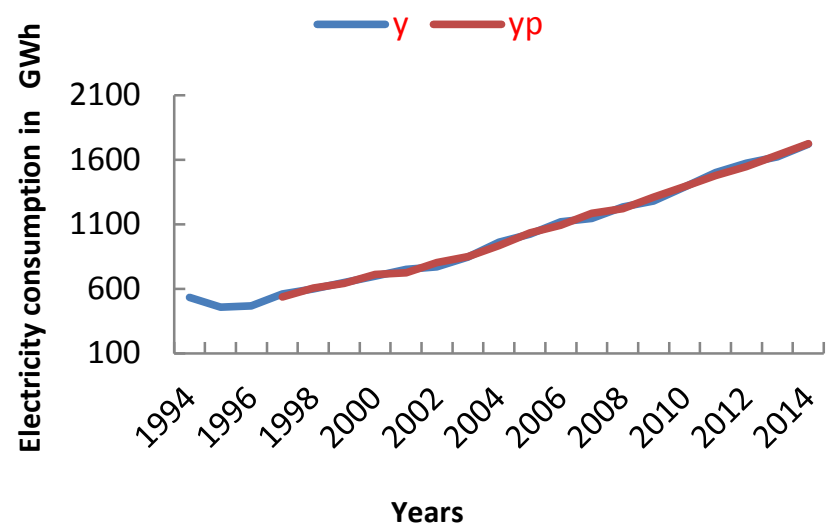

Figure 3. Evolution of fitted and real values of electricity consumption.

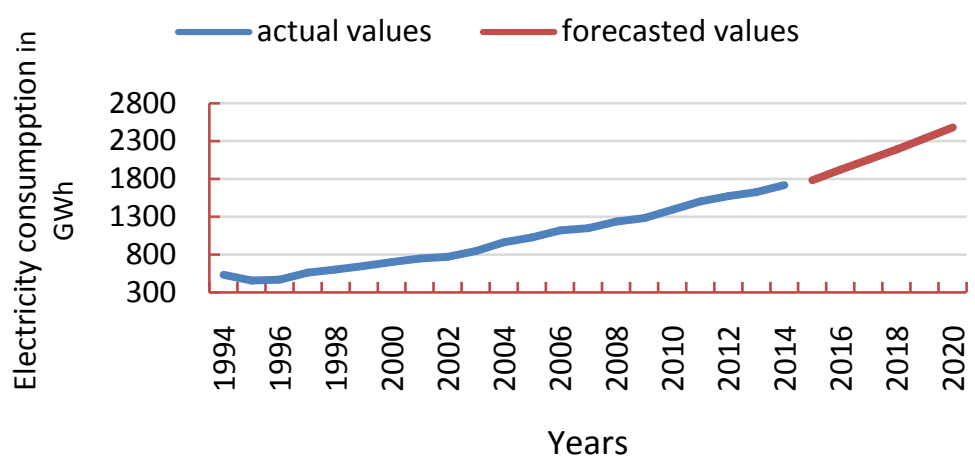

Figure 4. Residential electricity demand tendency. 


\section{Conclusions}

This work aimed at making an effective and reliable forecasting of the residential sector for electricity demand in Cameroon following a multilinear approach. This was in order to give new lights to the decision makers of the electricity sector and to guide their decisions.

A modeling is carried out via the EViews Software on the basis of VAR(1) model and allows to give characteristics of this demand.

Thus, when the demand in year $t-1$ increases a unit, that of the year $t$ increases of 7.42\%; when GDP per capita of the previous year grows of a unit, the consumption of the following year undergoes a rise of $127.17 \%$. And when the population, the number of households and subscribers grow accordingly of one unit in year $t-1$, electricity consumption evolves of $0.027 \%, 9.04 \%$ and $43.83 \%$ respectively at year $t$. All the selected determinants tend to carry the increase of the electric demand. The forecasted values confirm such an observation. The residential electricity consumption will skip from 1721 GWh in 2014 with a little more than $2481 \mathrm{GWh}$ in 2020 approximately. The residential demand will then be intensified in the coming years, the supply-demand gap will hollow out more and the deficit will slow down the economic growth. Decision makers of the electricity sector thus foresee a lightening horizon in order to make a better appreciation on the demand relating to that sector, and take all the possible measures so as to give it satisfaction. All the ongoing hydroelectric and thermal project should follow a speed up to that end.

\section{Conflicts of Interest}

The authors declare no conflicts of interest regarding the publication of this paper.

\section{References}

[1] Energy for Development (2013) Development of a National Energy Efficiency Policy, Strategy and Action Plan in Cameroon inception. Report 2013. https://www.euei-pdf.org

[2] Aydin, G. (2015) The Modeling and projection of primary Energy Consumption by the Sources. Energy Sources, Part B: Planning, and Policy, 10, 67-74. https://doi.org/10.1080/15567249.2013.771716

[3] Tamba, J.G., Nsouandélé, J.L., FopahLélé, A. and Sapnken, F.E. (2017) Electricity Consumption and Economic Growth: Evidence from Cameroon. Energy Sources, Part B: Economics, Planning, and Policy, 12, 1007-1014. https://doi.org/10.1080/15567249.2017.1349211

[4] Tatietse, T.T., Kemajou, A. and Diboma, B.S. (2015) Offre d'électricité et développement des entreprises industrielles au Cameroun. Mai, Yaoundé. https://fr.scribd.com/

[5] Muh, E., Amara, S. and Tabet, F. (2017) Sustainable Energy Policies in Cameroon: A Holistic Overview. Renewable and Sustainable Energy Reviews, 82, 3420-3429. https://doi.org/10.1016/j.rser.2017.10.049

[6] Fondja Wandji, Y.D. (2013) Energy Consumption and Economic Growth: Evidence 
from Cameroon. Energy Policy, 61, 1295-1304.

https://doi.org/10.1016/j.enpol.2013.05.115

[7] Maxime, K.K. (2006) Privatisation et bien être social: le cas de l'électricité au Cameroun. Thèse de doctorat, Université de Yaoundé II, Cameroun.

http://www.reseau-cicle.org

[8] Fotsing, F.I.M., Donatien, N. and Réné, T. (2014) Analysis of Demand and Supply of Electricity Energy in Cameroon: Influence of Meteorological Parameters on the Monthly Power Peak of South and North Interconnected Electricity Networks. International Journal of Energy and Engineering, 4, 168-185.

https://doi.org/10.11648/j.ijepe.20140304.12

[9] Fotsing, F.I.M., Donatien, N. and Réné, T. (2014) Influence of Socio-Economic Indicators on Electricity Consumption of Low Voltage Customers in Cameroon. International Journal of Energy and power Engineering, 34, 186-203.

https://doi.org/10.11648/j.ijepe.20140304.13

[10] Muh, E. and Tabet, F. (2018) Comparative Analysis of Hybrid Renewable Energy for Off-Grid Applications in Southern Cameroons. Renewable Energy, 135, 41-54. https://doi.org/10.1016/j.renene.2018.11.105

[11] Bourbonnais, R. (2009) Econométrie, manuel et exercice corrigés. Dunod, 7ème edition. https://www.dunod.com

[12] Ghoch, S. (2002) Electricity Consumption and Economic Growth in India. Energy Policy, 30, 125-129. https://doi.org/10.1016/S0301-4215(01)00078-7

[13] Energy of Cameroon Data Base (2014) https://www.sas.com

[14] World Bank Data Base (2014) https://data.worldbank.org

[15] Electricity Sector Regulatory Agency (2014) Development of a National Policy, an Energy Efficiency Action plan in Cameroon. https://www.euei-pdf.org 